\title{
Avaliação da qualidade de vida de mulheres com HIV/AIDS através do HAT-QoL
}

\author{
Evaluation of quality of life among women \\ with HIV/AIDS using HAT-QoL
}

\author{
1 Universidade Federal \\ do Ceará, Fortaleza, Brasil. \\ 2 Faculdade de Medicina \\ de Botucatu, Universidade \\ Estadual Paulista, \\ Botucatu, Brasil. \\ Correspondência \\ Marli Teresinha \\ Gimeniz Galvão \\ Rua Marcos Macedo \\ 1350/702, Fortaleza,CE \\ 60150-190, Brasil. \\ marli@ufc.br
}

\begin{abstract}
In order to evaluate quality of life among women with HIVIAIDS, the HIV-AIDS Quality of Life test (HAT-QoL) was administered as a specific tool for individuals infected with HIV. It consists of 42 questions and is divided into nine modules with questions on different aspects of the lives of persons with HIVIAIDS. The scale was subjected to reverse and pre-test translation. Its final form was applied to 73 women with HIVIAIDS. The results, following statistical analysis, indicated that the most negatively affected modules were: "Financial Concerns", "Concerns about Confidentiality”, "Sexual Activity”, and "Concerns with Health". These results were attributed to the fact that women demonstrated a significant socioeconomic disadvantage which (probably in addition to the infection itself) led to the negative impact on quality of life. It was concluded that the HAT-QoL scale was appropriate for the population of female HIV carriers in this study (despite having originated from a non-Brazilian culture), since application of the scale produced results similar to those found in international publications.
\end{abstract}

Quality of Life; Acquired Immunodeficiency Syndrome; Women's Health
Marli Teresinha Gimeniz Galvão 1

Ana Teresa de Abreu Ramos Cerqueira 2

Jussara Marcondes-Machado 2

\section{Introdução}

Antes dos anos 90, havia pouca solução terapêutica para arrefecer os efeitos da ação do HIV no indivíduo. Assim, os pacientes vivenciavam sua evolução clínica e esperavam a morte, sem expectativas alentadoras de tratamento. Apenas no início da década de 90 é que surgiram as primeiras tentativas de terapêuticas mais eficazes, o que trouxe maior esperança para os infectados.

Dessa forma, os portadores de HIV/AIDS tiveram ampliada não apenas sua expectativa de vida, como também a qualidade de vida, devido principalmente aos avanços terapêuticos, até que a cura ou vacina possam se tornar disponíveis.

Durante a última década, os instrumentos de medida de qualidade de vida foram sofrendo evolução. A maioria deles surgiu em decorrência da reformulação de instrumentos anteriores, reelaborados baseando-se mais no ponto de vista dos investigadores, do que de características da própria população a ser avaliada 1 .

Os estudos da qualidade de vida em pacientes portadores de HIV/AIDS têm utilizado instrumentos genéricos ou construídos para avaliação de outras doenças crônicas, como, por exemplo, o câncer, ou instrumentos adaptados para portadores de HIV/AIDS 2,3. Os instrumentos genéricos não observam questões importantes no contexto da vida cotidiana do porta- 
dor do HIV, como questões relativas à adaptação à doença, a sexualidade e ao relacionamento interpessoal. E, ainda, por terem sido adaptados de instrumentos utilizados em outras doenças, não consideram todo o drama social a que esta população de indivíduos portadores de HIV está exposta no decorrer da sua doença, como estigmas, preconceito e segregação 4.

Já o HIVIAIDS - Quality of Life (HAT-QoL), desenvolvido por Holmes \& Shea 5, é um instrumento construído especificamente a indivíduos infectados pelo HIV. Apresenta 42 questões e é dividido em nove domínios: atividade geral (oito itens), atividade sexual (três itens), preocupação com sigilo sobre a infecção (cinco itens), preocupação com a saúde (cinco itens), preocupação financeira (quatro itens), conscientização sobre o HIV (três itens), satisfação com a vida (oito itens), questões relativas à medicação (quatro itens) e confiança no médico (três itens).

A qualidade de vida das pessoas com HIV/ AIDS tem sido uma preocupação importante nas pesquisas da área. Desde a introdução do AZT (zidovudina) em 1986, têm sido realizados ensaios clínicos destinados a avaliar a eficácia dos esquemas de tratamentos nos pacientes portadores de HIV, procurando focalizar inclusive a qualidade de vida 6 . No entanto, estes estudos têm sido desenvolvidos principalmente entre homens, provavelmente porque, ou as mulheres chegam mais tarde aos serviços de saúde, ou não são diagnosticadas. Além disso, as pesquisas clínicas e terapêuticas excluem as mulheres devido ao elevado potencial de risco fetal 7,8. Por estes motivos, estudos que tratam da qualidade de vida entre as mulheres são raros 9,10, o que fundamentou a presente pesquisa.

\section{Objetivo}

Avaliar em mulheres com infecção pelo HIV ou com AIDS, a qualidade de vida, por meio da aplicação da escala HAT-QoL.

\section{Metodologia}

O HAT-QoL foi aplicado em 73 mulheres com infecção pelo HIV ou com AIDS, não as diferenciando, uma vez que em estudo prévio não se constatou diferença estatisticamente significante entre esses dois grupos no que se referia à qualidade de vida 11 . O estudo foi conduzido em ambulatório especializado no atendimento a pacientes com HIV/AIDS de uma universidade pública do Estado de São Paulo, Brasil, no período de dezembro de 2000 a abril de 2001.

Para seleção das pacientes adotou-se como critérios de inclusão ser mulher com idade igual ou superior a 18 anos, apresentar sorologia positiva para o HIV e aceitar participar da pesquisa. Como critério de exclusão: presença de doença mental e qualquer outro fator que impedisse ou interferisse na entrevista. Não houve exclusão e nem recusa de nenhuma das mulheres convidadas a participar da pesquisa.

Para aplicação do HAT-QoL utilizou-se de entrevista individualizada em sala privativa sem a presença de outros indivíduos que pudessem interferir nas respostas.

O estudo foi avaliado e aprovado por Comitê de Ética em Pesquisa. Todas as pacientes foram devidamente esclarecidas sobre a investigação e assinaram o Termo de Consentimento Livre e Esclarecido, seguindo as exigências para pesquisa que envolvem seres humanos, de acordo com a Resolução no 196 de 1996 do Conselho Nacional de Ética em Pesquisa 12.

Para coleta de dados utilizou-se instrumento estruturado que permitia a caracterização das mulheres e a aplicação da escala de medida de qualidade de vida HAT-QoL.

O instrumento HAT-QoL foi submetido ao procedimento de tradução reversa. Foi traduzido e retraduzido por dois profissionais com proficiência na língua inglesa; a língua materna de um era a portuguesa e a do outro, a inglesa. Ambos foram orientados a traduzir atentamente as interpretações divergentes de itens ambíguos do texto original. Não foi mantido qualquer contato entre os profissionais por ocasião da tradução, obtendo-se, portanto, dois textos independentes. Foi realizada revisão pelo autor e pelos tradutores do instrumento, para ajustes da tradução. A seguir, foi aplicado o instrumento como pré-teste, realizando-se, a seguir, uma segunda revisão para adequar o instrumento ao nível de entendimento das pacientes.

O questionário original foi mantido em sua estrutura quanto à formulação das questões, uma vez que nenhuma delas referia-se a experiências estranhas à realidade das pacientes a serem avaliadas. A versão em português (Tabela 1) foi então aplicada a cinco pacientes portadoras de HIV, atendidas ambulatorialmente e, tendo-se obtido resultado satisfatório, foi aplicada na população definitiva do estudo. 


\section{Domínio 1: Atividades gerais}

1. Nas últimas quatro semanas, fiquei satisfeita com a minha atividade física. *

2. Nas últimas quatro semanas, fiquei limitada fisicamente na minha habilidade de realizar os afazeres de casa.

3. Nas últimas quatro semanas, a dor tem limitado minha habilidade de ser fisicamente ativa.

4. Nas últimas quatro semanas, fiquei preocupada por não ser capaz de ir ao serviço ou cumprir as atividades rotineiras do dia-a-dia como costumava fazer no passado.

5. Nas últimas quatro semanas, tenho sentido que portar o HIV tem limitado a quantidade de trabalho que consigo fazer no serviço ou nas minhas atividades rotineiras do dia-a-dia.

6. Nas últimas quatro semanas, tenho me sentido cansada demais para ser socialmente ativa.

7. Nas últimas quatro semanas, minha saúde tem limitado minhas atividades sociais.

\section{Domínio 2: Atividades sexuais}

1. Nas últimas quatro semanas, fiquei satisfeita com minha vida sexual.*

2. Nas últimas quatro semanas, estive interessada em sexo.*

3. Nas últimas quatro semanas, foi difícil para mim ficar excitada sexualmente.

\section{Domínio 3: Preocupação com o sigilo sobre a infecção}

1. Nas últimas quatro semanas, limitei o que conto para os outros sobre mim mesma.

2. Nas últimas quatro semanas, tive medo de contar para os outros que estou com o HIV.

3. Nas últimas quatro semanas, fiquei preocupada a respeito dos meus parentes descobrirem que estou com o HIV.

4. Nas últimas quatro semanas, fiquei preocupada a respeito das pessoas no meu serviço ou atividades rotineiras do dia-a-dia descobrirem que estou com o HIV

5. Nas últimas quatro semanas, fiquei preocupada pensando que poderia perder minha fonte de renda se outras pessoas descobrirem que estou com o HIV.

\section{Domínio 4: Preocupações com a saúde}

1. Nas últimas quatro semanas, não consegui viver como gostaria porque estou tão preocupada com a minha saúde.

2. Nas últimas quatro semanas, fiquei preocupada pensando que a minha saúde poderia piorar.

3. Nas últimas quatro semanas, fiquei preocupada a respeito da minha contagem de células CD4.

4. Nas últimas quatro semanas, senti incerteza a respeito do que o futuro me reserva.

5. Nas últimas quatro semanas, me preocupei pensando em quando irei morrer.

\section{Domínio 5: Preocupações financeiras}

1. Nas últimas quatro semanas, me preocupei a respeito de ter de viver com renda fixa.

2. Nas últimas quatro semanas, me preocupei a respeito de como irei pagar minhas contas.

3. Nas últimas quatro semanas, o dinheiro foi muito pouco para eu me cuidar como acho que deveria.

4. Nas últimas quatro semanas, tive dinheiro suficiente para fazer as coisas que gosto de fazer.*

\section{Domínio 6: Conscientização sobre o HIV}

1. Nas últimas quatro semanas, me arrependi a respeito de como vivi a minha vida antes de descobrir que estava com o HIV

2. Nas últimas quatro semanas, senti raiva a respeito do meu comportamento de risco para o HIV no passado.

3. Nas últimas quatro semanas, consegui aceitar o fato de que estou com o HIV.*

\section{Domínio 7: Satisfação com a vida}

1. Nas últimas quatro semanas, aproveitei a vida. ${ }^{\star}$

2. Nas últimas quatro semanas, senti uma forte vontade de viver.*

3. Nas últimas quatro semanas, me senti satisfeita com a minha vida.*

4. Nas últimas quatro semanas, me senti em controle da minha vida.*

5. Nas últimas quatro semanas, me senti bem comigo mesma. *

6. Nas últimas quatro semanas, me senti motivada a fazer as coisas.*

7. Nas últimas quatro semanas, fiquei satisfeita em ver como sou socialmente ativa.*

8. Nas últimas quatro semanas, me senti contente em ver como tenho estado saudável.*

(continua) 


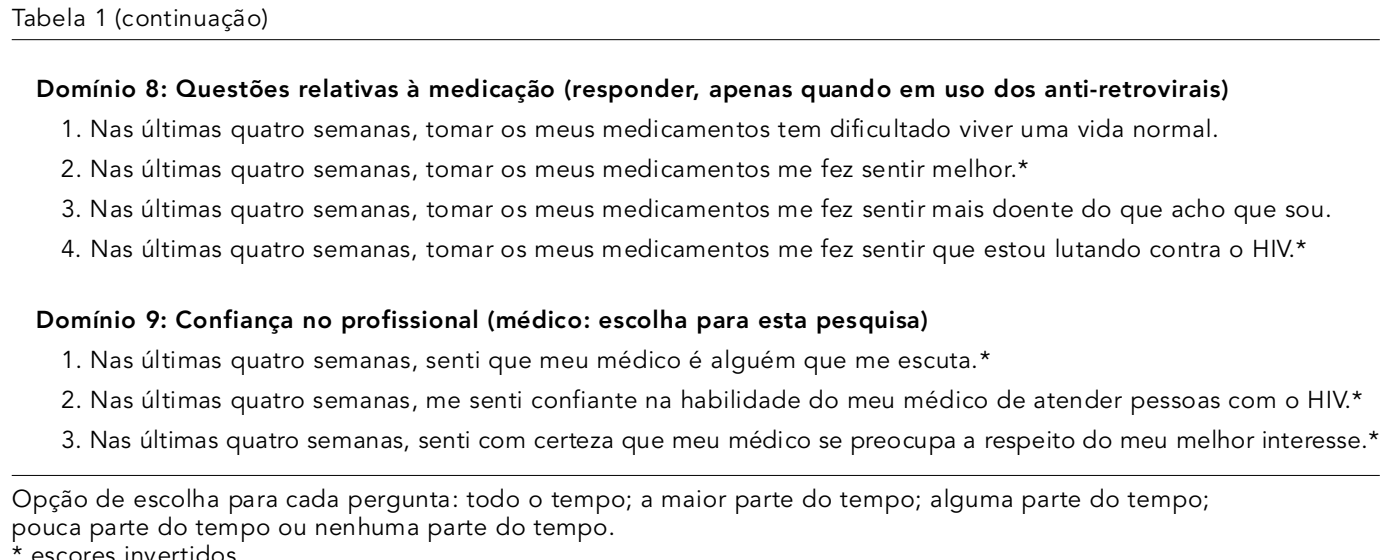

Para cada item da escala havia cinco opções de escolha, entretanto a paciente deveria escolher apenas uma alternativa que apresentava pontuação que variava de um a cinco, sendo que um corresponde ao pior estado e cinco ao melhor estado ou condição.

Para análise de dados, os escores foram transformados em índices com ponderação de 0 a 100 , em que 0 corresponde ao menor e 100 ao maior valor encontrado para cada domínio.

Considerou-se com melhor qualidade de vida aquelas mulheres que apresentaram maiores índices e, em piores, aquelas com menores índices obtidos. Neste estudo, considerou-se como prejudicados os domínios que apresentaram índices cuja média e mediana foram inferiores a 55 pontos, uma vez que a pontuação máxima poderia ser de cem pontos.

Os dados foram digitados e analisados no programa estatístico SPSS-8.0. Para descrever os índices dos domínios da escala de qualidade de vida empregaram-se a média e o desvio padrão. Utilizou-se para análise de normalidade o Teste de Kolmogorov-Smirnov 13.

Para o estudo das correlações entre os índices dos diferentes domínios foram utilizados os coeficientes de correlação de Pearson para as variáveis cuja distribuição obedeceu à lei de normalidade, e o de Spearman para as variáveis cuja distribuição não obedeceu à lei de distribuição da normalidade.

\section{Resultados e discussão}

As mulheres estudadas tinham idades entre 19 e 56 anos, $60,3 \%$ eram casadas ou moravam junto com o parceiro e $50 \%$ apresentavam até oito anos de escolaridade. Do total, $64,3 \%$ conviviam com renda per capita menor que um salário mínimo, equilavente a $\mathrm{R} \$ 151,00$ (US\$60,00) na época do estudo. Quase a totalidade das mulheres foi infectada por relações heterossexuais.

$\mathrm{Na}$ análise dos índices mais baixos da escala de qualidade de vida, os resultados demonstraram que os domínios mais comprometidos foram: "Preocupações financeiras", "Preocupação com sigilo sobre a infecção", "Atividades sexuais" e "Preocupações com a saúde" (Tabela 1).

Provavelmente, o prejuízo na qualidade de vida, no que se refere a "Preocupações financeiras", é resultado de uma situação econômica que dificulta a sobrevivência do indivíduo. O índice médio obtido $(31,3)$ nesse domínio, provavelmente decorreu da baixa renda per capita observada entre as mulheres, com média de $\mathrm{R} \$ 151,00$, equivalente a um salário mínimo vigente à época da obtenção dos dados (Tabela 2).

Esse dado pode indicar que a renda, além de ter o seu valor ligado às condições e serviços de sua região, pode ser decorrente do estado de saúde e do estado funcional do indivíduo, o que dificultaria ainda mais sua inserção no mercado de trabalho. Dessa forma, o baixo rendimento per capita pode ter sido um fator que levou as mulheres a apresentarem baixos índices neste domínio.

Cruz \& Brito 14, ao atenderem grupos de mulheres portadoras de HIV em organização não-governamental do Estado de São Paulo, observaram que a qualidade de vida dessas mulheres ficava atrelada a condições financeiras melhores, que facilitariam o autocuidado e o tratamento. Diante destes fatos, a qualidade de vida está intimamente ligada à inserção socioeconômica e à exclusão social.

Outro domínio a apresentar índice médio abaixo de $50(39,2)$ foi "Preocupação com sigilo sobre a infecção", o que sugere comprometimento de qualidade de vida no que se refere a ele (Tabela 2).

A preocupação com a revelação de estar infectado, muitas vezes é auto-imposta pelo me- 
Valores descritivos e nível descritivo $(p)$ do teste de normalidade dos índices dos domínios da escala de qualidade de vida HAT-QoL, entre as 73 mulheres com infecção pelo HIV/AIDS.

\begin{tabular}{|c|c|c|c|c|c|c|c|c|c|c|}
\hline Domínios & Itens & $\mathbf{n}^{\star \star}$ & Média & DP & Mínimo & P25 & Mediana & P75 & Máximo & $p$ \\
\hline Atividades gerais * & 7 & 73 & 77,0 & 22,8 & 10,7 & 66,1 & 85,7 & 92,9 & 100,0 & 0,0384 \\
\hline Atividade sexual & 3 & 73 & 52,5 & 26,0 & 0,0 & 33,3 & 50,0 & 70,8 & 100,0 & 0,0662 \\
\hline Preocupação com o sigilo & 5 & 73 & 39,2 & 30,3 & 0,0 & 15,0 & 40,0 & 60,0 & 100,0 & 0,0538 \\
\hline Preocupação com a saúde & 5 & 73 & 52,7 & 29,6 & 0,0 & 25,0 & 55,0 & 80,0 & 100,0 & 0,5224 \\
\hline Preocupação financeira* & 4 & 73 & 31,3 & 27,4 & 0,0 & 12,5 & 25,0 & 50,0 & 100,0 & 0,0006 \\
\hline Conscientização sobre o HIV & 3 & 73 & 63,0 & 28,3 & 0,0 & 37,5 & 66,7 & 87,5 & 100,0 & 0,2125 \\
\hline Satisfação com a vida & 8 & 73 & 75,5 & 19,2 & 25,0 & 62,5 & 78,1 & 93,8 & 100,0 & 0,2800 \\
\hline Questões relativas à medicação & 4 & 61 & 76,5 & 19,6 & 25,0 & 62,5 & 75,0 & 93,8 & 100,0 & 0,3240 \\
\hline Confiança no médico* & 3 & 73 & 91,6 & 19,9 & 0,0 & 95,8 & 100,0 & 100,0 & 100,0 & $<0,0001$ \\
\hline
\end{tabular}

* Não segue a lei da distribuição Normal $(p<0,05)$.

** Número de pacientes.

$\mathrm{DP}=$ desvio padrão; P25 = percentil 25; P75 = percentil 75.

do que o portador ou doente, tem de, ao tornar conhecido seu diagnóstico, ficar sujeito a preconceitos e estigmatização.

Lent \& Valle 15 foram categóricos ao referir que uma grande parte dos indivíduos soropositivos esconde o fato de estarem contaminados, pelo maior tempo e da melhor maneira possível, ingressando na clandestinidade quanto à sua condição. Com isso, tenta driblar o isolamento social ao qual poderia estar submetido, inevitavelmente, na maioria das vezes, caso se declarasse portador do HIV.

Além disso, as experiências vivenciadas e acumuladas pela autora, advindas da assistência direta à população estudada, estão de acordo com a referência de Mello 16, que constatou que trabalhadoras portadoras, freqüentemente são despedidas, ou não são contratadas, pois, para o controle médico de seu estado de portador, necessitam ausentar-se rotineiramente do trabalho, ora para consultas, ora para exames, ou ainda, para obtenção de medicamentos. Dessa forma, o sigilo é uma opção imposta.

A autora, a partir ainda de sua assistência direta a essas pacientes, também pôde observar que as mulheres, para não denunciar a sua doença, utilizam-se de subterfúgios: escondem frascos de medicações anti-retrovirais ou trocam suas embalagens; às vezes falam de sua infecção como sendo outra doença; às vezes buscam tratamentos em municípios distantes, com medo de serem reconhecidas.

Pereira 17 refere que durante $o$ atendimento à mulher portadora de HIV, observa-se que elas experimentam diversos sentimentos relacionados ao seu estado. Não entrar em contato com a verdade sobre seu diagnóstico, muitas vezes é uma forma de evitar sofrimento. Não relatar sua doença é mais uma maneira de não se identificar, mantendo um sigilo, muitas vezes até para consigo mesma.

Outros pesquisadores apontam que recurso utilizado pelos indivíduos de manter oculta sua contaminação, também serve para proteger os familiares e filhos da rejeição e discriminação que possam sofrer 4,18 .

Flaskerud 19 refere que, entre os pacientes, o maior estresse ocorreu no momento do conhecimento do diagnóstico de AIDS, persistindo durante todo o curso da doença; assim, a situação do segredo é um fato que, freqüentemente, coloca o paciente em hipervigilância. Muitos omitem a doença da família, dos amigos e até dos próprios parceiros.

Ao contrário, nos períodos que antecederam a era dos anti-retrovirais, o relato do diagnóstico à família e amigos não era uma questão de escolha, mas sim de necessidade, pois as conseqüências clínicas das doenças oportunistas eram rápidas e a debilitação física evidente. Dessa forma, os pacientes eram obrigados a falar com mais freqüência de sua doença do que hoje.

O domínio “Atividades gerais” correlacionou-se com os domínios "Satisfação com a vida" e "Confiança no profissional" (Tabela 3). A correlação entre os dois primeiros sugere que as mulheres que tinham pior satisfação tinham também comprometidas as condições físicas. A correlação destes dois com a "Confiança no profissional” também pode ser entendida, pois o médico é aquele que pode levar o doente a se manter fisicamente bem, para desenvolver suas atividades e, portanto, ter satisfação com a vida. 
Correlação entre os índices dos domínios da escala de qualidade de vida HAT-QoL, obtidos das 73 mulheres com infecção pelo HIV/AIDS.

\begin{tabular}{|c|c|c|c|c|c|c|c|c|}
\hline Índices & $\begin{array}{l}\text { Atividade } \\
\text { sexual }\end{array}$ & $\begin{array}{l}\text { Preocupação } \\
\text { com o sigilo }\end{array}$ & $\begin{array}{l}\text { Preocupação } \\
\text { com a saúde }\end{array}$ & $\begin{array}{l}\text { Preocupação } \\
\text { financeira }\end{array}$ & $\begin{array}{l}\text { Conscien- } \\
\text { tização } \\
\text { sobre o HIV }\end{array}$ & $\begin{array}{l}\text { Satisfação } \\
\text { com a vida }\end{array}$ & $\begin{array}{l}\text { Questões } \\
\text { relativas à } \\
\text { medicação }\end{array}$ & $\begin{array}{l}\text { Confiança } \\
\text { no médico }\end{array}$ \\
\hline Atividades gerais & 0,2146 & 0,0539 & 0,1227 & 0,0021 & 0,0940 & $0,4841^{\star \star}$ & 0,0976 & $0,3364^{\star *}$ \\
\hline Atividade sexual & 1,0000 & $-0,1222$ & 0,1987 & 0,1537 & $0,2865^{\star}$ & $0,3521 * *$ & $-0,0012$ & 0,1914 \\
\hline Preocupação com o sigilo & - & 1,0000 & $0,3219 * \star$ & 0,1307 & 0,0914 & $0,2828^{\star}$ & 0,1740 & 0,2293 \\
\hline Preocupação com a saúde & - & - & 1,0000 & $0,3437 * \star$ & $0,3003^{\star \star}$ & $0,4167^{\star \star}$ & 0,1109 & 0,2053 \\
\hline Preocupação financeira & - & - & - & 1,0000 & 0,2156 & $0,2580 *$ & 0,1689 & $-0,0556$ \\
\hline Conscientização sobre o HIV & - & - & - & - & 1,0000 & 0,2232 & 0,1597 & 0,0514 \\
\hline Satisfação com a vida & - & - & - & - & - & 1,0000 & $0,2759 *$ & $0,4882^{\star \star}$ \\
\hline $\begin{array}{l}\text { Questões relativas } \\
\text { à medicação }\end{array}$ & - & - & - & - & - & - & 1,0000 & $-0,0299$ \\
\hline Confiança no médico & - & - & - & - & - & - & - & 1,0000 \\
\hline
\end{tabular}

$\star p<0,05$.

$\star \star *<<0,01$

Diversos autores referem que a confiança no médico é uma situação de demonstração da fé que o paciente deposita no profissional, motivado por um relacionamento terapêutico afinado.

Bakken et al. 20 referem que uma efetiva relação médico-paciente tem influenciado na evolução da doença, associando-se com melhor condição de saúde e maior nível de adesão à terapêutica. Esse relacionamento próximo pode ter sido um fator que contribuiu na correlação acima citada, e que, ainda pode ter conduzido as mulheres a apresentarem mais satisfação com a vida e melhores condições no domínio “Atividades gerais".

Quanto ao domínio "Atividades sexuais", que investiga o interesse, a motivação para a relação sexual, encontrou-se índice médio de 52,5; fato que indica comprometimento na sua qualidade de vida nesse aspecto (Tabela 2). Vários pesquisadores relatam que as mulheres, ao tomarem conhecimento de seu diagnóstico, experimentam conflitos relacionados à sexualidade, com período de diminuição das atividades sexuais 17,21. Dessa forma, esses autores reforçam que a AIDS impõe mudanças nas atividades sexuais, e que as condições sob as quais se encontram devido à infecção, as levam a ter medo do relacionamento sexual, fazendo com que se esquivem das relações, mesmo apresentando desejo sexual.

Também é apontado que, pelo fato de as mulheres terem sido, quase exclusivamente, contaminadas pela via sexual, passam a sentir medo, constrangimento, abstendo-se das relações sexuais 17,21 .

O domínio "Atividades sexuais", correlacionou-se com os domínios "Conscientização so- bre o HIV" e "Satisfação com a vida" (Tabela 3). É provável que estes resultados sejam decorrentes do fato de as mulheres da presente pesquisa terem sido, na sua grande maioria, contaminadas exclusivamente pela via sexual. Isto pode indicar que, muitas vezes, as mulheres percebem que seu risco advém do comportamento de seus parceiros, usuários de drogas ilícitas e/ou com múltiplas parcerias sexuais. Muitas mulheres, após saberem de seu diagnóstico, referiram que já tinham conhecimento do risco de virem a se contaminar por seu parceiro, o que lhes causava raiva e culpa.

O prejuízo no domínio "Satisfação com a vi$d a$ " (Tabela 2) pode ser a expressão da falta de controle das mulheres sobre a própria vida. Eram, em sua maioria, dependentes exclusivamente dos parceiros para manutenção do lar, viviam com escassos recursos financeiros, sem apoio, sem trabalho, o que as fazia aceitar a situação de conviver em ambiente pouco favorável para suas aspirações. Esta situação pode ter comprometido a qualidade de vida das mulheres nas questões relacionadas à satisfação com a vida.

Os domínios "Atividades sexuais", "Conscientização sobre o HIV" e "Satisfação com a vi$d a$ " (Tabela 3) se correlacionaram, sugerindo que os processos de reavaliação, efetuados pelas mulheres após o diagnóstico, podem ter contribuído para o comprometimento de sua qualidade de vida.

O domínio "Preocupações com a saúde", que investiga a inquietação das mulheres em viver com o vírus e suas conseqüências, apresentou baixos índices (Tabela 2). Provavelmente, devido ao fato de que as situações investi- 
gadas nesse domínio se referiram à auto-avaliação em relação à vigilância da saúde, ao conhecimento de parâmetros laboratoriais, à autopercepção da saúde e à preocupação com um prognóstico de morte, preocupações que possivelmente impediam o bem-estar. Sob essa perspectiva, a preocupação com a saúde deveria ser uma situação esperada e desejável no indivíduo portador da infecção pelo HIV, porém, no presente estudo essa preocupação foi considerada como um prejuízo à qualidade de vida provavelmente pelo peso que representava. Entretanto, entre as portadoras, seria necessário ampliar as informações e os cuidados utilizando-se uma metodologia que não interferisse na "preocupação com a saúde". Talvez, este achado também se deva às condições de vida das mulheres e à forma como elas administram suas vidas, pois pode ser que, embora conheçam formas e maneiras de se manter "mais saudável”, suas condições clínicas e sociais não permitam que as executem.

\section{Considerações}

O instrumento utilizado neste estudo para avaliação de qualidade de vida entre mulheres com infecção pelo HIV, demonstrou importante comprometimento na qualidade de vida dessas mulheres, talvez por conviverem com situações que as conduzem à precariedade

\section{Resumo}

Com objetivo de avaliar qualidade de vida entre mulheres portadoras de HIVIAIDS, aplicou-se o HIVIAIDS - Quality of Life test (HAT-QoL), instrumento específico para indivíduos infectados pelo HIV. Compõe-se de 42 questões e divide-se em nove domínios com questões sobre diversos aspectos de vida dos portadores. A escala foi submetida à tradução reversa e pré-teste. Sua forma definitiva foi aplicada entre 73 mulheres com HIVIAIDS. Os resultados, após análise estatística, indicaram que os domínios mais comprometidos foram: "Preocupações financeiras", "Preocupação com sigilo sobre a infecção", "Atividades sexuais" $e$ "Preocupações com a saúde". Esses resultados foram atribuídos ao fato das mulheres apresentarem, além da infecção, uma situação sócio-econômica precária que, provavelmente, também interferiu negativamente em sua qualidade de vida. Concluímos que a escala HATQoL foi adequada para a população de mulheres portadoras de HIV deste estudo, apesar de ser originária de uma cultura diferente da brasileira, já que sua aplicação produziu resultados semelhantes aos da literatura internacional.

Qualidade de Vida; Síndrome de Imunodeficiência Adquirida; Saúde da Mulher econômica e social. Os domínios da escala de qualidade de vida HAT-QoL, cujos índices apontaram prejuízo na qualidade de vida das mulheres infectadas pelo HIV foram: "Preocupações financeiras", "Preocupações com a saúde", "Preocupação com o sigilo sobre a infecção" e "Atividades sexuais".

A escala HAT-QoL demonstrou ser útil para a população de mulheres portadoras de HIV deste estudo, apesar de ser originária de uma cultura diferente da brasileira, já que sua aplicação produziu resultados semelhantes aos da literatura internacional.

Diversos estudos conduzidos no mundo apontam que a qualidade de vida dos pacientes com infecção pelo HIV vem sendo substancialmente modificada a partir da instituição das novas drogas para tratamento específico. As mulheres da presente pesquisa apesar de receberem o tratamento específico para a infecção pelo HIV, apresentam comprometimento de sua qualidade de vida, talvez pelas adversidades sócio-econômicas e impostas pelo meio social em que vivem, além da infecção.

Depreende-se que ao viver com HIV as mulheres deparam-se com situações de discriminação, segregação, falta de recursos sociais e financeiros, ruptura nas relações afetivas e problemas com a sexualidade. Diante deste panorama, viver e enfrentar a doença torna-se cada vez mais problemático e, como conseqüência, a qualidade de vida pode ser comprometida.

\section{Colaboradores}

M. T. G. Galvão participou na revisão de literatura, elaboração do projeto, coleta de dados, análise dos resultados e redação do artigo final. A. T. A. R. Cerqueira contribuiu na orientação metodológica, análise dos resultados e redação final do artigo. J. Marcondes-Machado colaborou com a orientação do projeto, análise dos resultados e redação do artigo final. 


\section{Referências}

1. Brown JS, Rawlinson ME, Hilles NC. Life satisfaction and chronic disease: exploration of a theorical model. Med Care 1986; 19:1136-46.

2. Carr AJ, Thompson PW, Kirman JR. Outcomes series. Quality of life measures. Br J Rheumatol 1996; 35:275-81.

3. Holmes WC, Shea JA. Two approaches to measuring quality of life in the HIV/AIDS populations: HAT-QoL and MOS-HIV. Qual Life Res 1999; 8:515-27.

4. Vignale R, Calandria L. Calidad de vida de los pacientes com VIH/SIDA. Bol Acad Nac Med B Aires 1999;77:385-9.

5. Holmes WC, Shea JA. A new HIV/AIDS-target quality of life (HAT-QoL) instrument: development, reliability, and validity. Med Care 1998; 36:138-54.

6. Badia X, Guerra L, Garcia M, Podzamczer D. La evaluación de la calidad de vida en los pacientes con infección por el VIH y sida. Med Clin 1999; 112:739-44

7. Vermelho LL, Barbosa RHS, Nogueira SA. Mulheres com AIDS: desvendando histórias de risco. Cad Saúde Pública 1999; 15:369-79.

8. Sarna L, Servellen GV, Padilha G, Brecht ML. Quality of life in women with symptomatic HIV/ AIDS. J Adv Nurs 1999; 30:597-605.

9. Hays RD, Cunningham WE, Sherbourne CD, Wilson IB, Wu AW. Quality of life in patients with human immunodeficiency virus. Am J Med 2000; 108:714-22.

10. Zorilla CD, Santiago LE. La mujer el VIH/SIDA: barreras y nuevos desafios. Public Report Health Science Journal 1999; 18:397-400.

11. Galvão MTG. Aplicação do instrumento do HATQoL para análise da qualidade de vida de mulheres com infecção pelo HIV ou com aids, e sua correlação com as variáveis sociodemográficas, epidemiológicas e clínicas [Tese de Doutorado]. Botucatu: Faculdade de Medicina de Botucatu, Universidade Estadual Paulista; 2002.
12. Ministério da Saúde. Manual operacional para Comitês de Ética em Pesquisa. Brasília: Ministério da Saúde; 2002.

13. Fisher LD, Belle GV. Biostastitics: a methodology for the health sciences. New York: Willey Interscience; 1993.

14. Cruz EF, Brito N. Fios da vida: tecendo o feminino em tempos de aids. Brasília: Ministério da Saúde. http:/ /www.aids.gov.br/biblioteca (acessado em 20/Mar/2000).

15. Lent C, Valle A. Aids: sujeito e comunidade. São Paulo: IDAC; 2000.

16. Mello MCPF. Contornos femininos da aids em São José do Rio Preto: Impasses e desafios [Dissertação de Mestrado]. Araraquara: Faculdade de Filosofia Ciências e Letras de Araraquara, Universidade Estadual Paulista; 1999.

17. Pereira MLD. A re(invenção) da sexualidade feminina após a infecção pelo HIV [Tese de Doutorado]. São Paulo: Escola de Enfermagem, Universidade de São Paulo; 2001.

18. Bunting S. Sources of stigma associated with women with HIV. Adv Nurs Sci 1996;19:64-73.

19. Flaskerud JH. Aspectos psicossociais e neuropsiquiátricos. In: Flaskerud JH, organizador. AIDS/ Infecção pelo HIV. Rio de Janeiro: Editora Medsi; 1992. p. 163-90.

20. Bakken S, Holzemer W, Brow MA, Powell-Cope GM, Turnes JG, Inouye J. Relationships between perception of engagement with health care provider and demographic characteristics, health status, and adherence to therapeutic regimen in persons with HIV/AIDS. AIDS Patient Care STDS 2000; 14:189-97.

21. Grimberg M. Género y VIH/SIDA. Un análisis de los diferenciales de género en la experiencia de vivir con VIH. Cuad Med Soc 2000; 78: 41-54.

Recebido em 09/Mai/2003

Versão final reapresentada em 19/Set/2003

Aprovado em 16/Out/2003 